\title{
Biomimetic Underwater Robots Based on Dielectric Elastomer Actuators
}

\author{
Jun Shintake ${ }^{1}$, Herbert Shea ${ }^{2}$, and Dario Floreano ${ }^{1}$
}

\begin{abstract}
Dielectric elastomer actuators (DEAs), a soft actuator technology, hold great promise for biomimetic underwater robots. The high-voltages required to drive DEAs can however make them challenging to use in water. This paper demonstrates a method to create DEA-based biomimetic swimming robots that operate reliably even in conductive liquids. We ensure the insulation of the high-voltage DEA electrodes without degrading actuation performance by laminating silicone layers. A fish and a jellyfish were fabricated and tested in water. The fish robot has a length of $120 \mathrm{~mm}$ and a mass of $3.8 \mathrm{~g}$. The jellyfish robot has a $61 \mathrm{~mm}$ diameter for a mass of $2.6 \mathrm{~g}$. The measured swimming speeds for a periodic $3 \mathrm{kV}$ drive voltage were $\sim 8 \mathrm{~mm} / \mathrm{s}$ for the fish robot, and $\sim 1.5 \mathrm{~mm} / \mathrm{s}$ for the jellyfish robot.
\end{abstract}

\section{INTRODUCTION}

Swimming robots can be used for underwater exploration, observation, inspection, and rescue. Biomimetics is a promising design approach for such robots, in view of the high mobility and efficiency of many aquatic animals [1]. One can also use biomimetic underwater robots as a tool to aid in understanding the underlying principles of animal locomotion [2], [3].

While electromagnetic motors are most widely used to actuate biomimetic underwater robots, several recent studies have focused on the use of soft actuators. One of the benefits of soft actuators is the resulting simplicity of the robot structure, as mechanical parts such as gears and linkages that are required in the traditional approach are not needed with compliant actuators. In addition, the compliance of the actuators provides a nearly infinite number of degrees of freedom, allowing mimicking the continuous deformations observed in animals. In this context, several soft actuator technologies such as ionic polymer-metal composites (IPMCs), lead zirconate titanates (PZTs), shape memory alloys (SMAs), and pneumatic elastomeric actuators, have been successfully demonstrated in biomimetic underwater robots [4], [5].

We use dielectric elastomer actuators (DEAs) [6]-[8] for biomimetic underwater robots because they combine many appealing features. DEAs exhibit high compliance $(\sim 1 \mathrm{MPa}$ of elastic modulus), large actuation strokes (e.g., $85 \%$ of linear strain [9]), fast response time (less than $200 \mu \mathrm{s}$

This work was supported by the Swiss National Centre of Competence in Research (NCCR) Robotics, and the Swiss National Science Foundation grant 200020-153122.

${ }^{1}$ J. Shintake and D. Floreano are with the Laboratory of Intelligent Systems (LIS), École Polytechnique Fédérale de Lausanne, Route Cantonale, Lausanne 1015, Switzerland. jun. shintakedepfl. ch

${ }^{2} \mathrm{H}$. Shea is with the Microsystems for Space Technologies Laboratory (LMTS), École Polytechnique Fédérale de Lausanne, Rue de la Maladière 71B, Neuchâtel 2000, Switzerland.

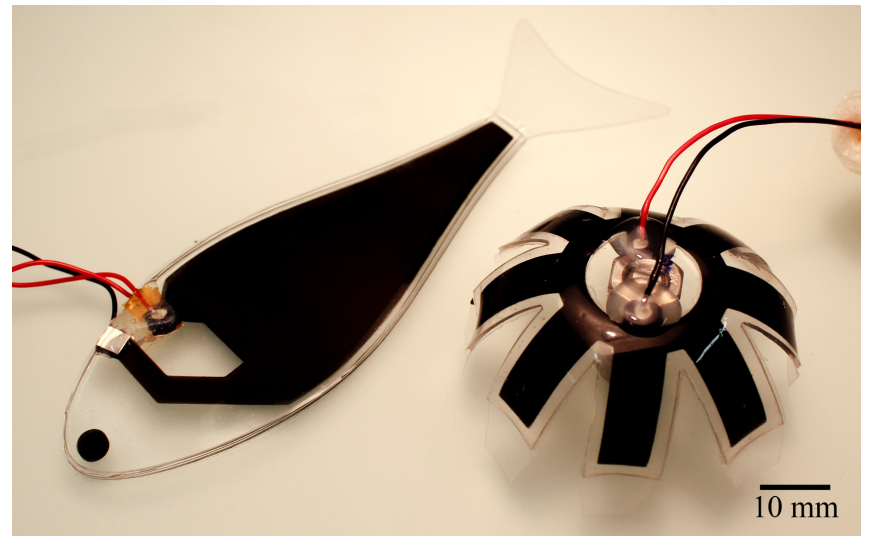

Fig. 1. Two DEA-based simple biomimetic underwater robots fabricated in this study: a fish robot (left) and a jellyfish robot (right).

[10]), and theoretically high electromechanical efficiency (max. $90 \%$ [6]). However, very little work is reported on DEA-based underwater robots. To our knowledge, only one attempt of a jellyfish type robot has been reported [11]. One possible reason is that DEAs typically require several $\mathrm{kV}$ for actuation, which requires excellent electrical insulation between the actuators as well as drive electronics (such as DC/DC converters) from the surrounding water. Coating the robot with electrically insulating material is bulky or can greatly stiffen the structure, thus limiting the inherent performance of the soft actuator.

This paper demonstrates a method to create simple DEAbased biomimetic underwater robots. The method consists of laminate soft silicone layers, ensuring the insulation of the high-voltage DEA electrodes, and is applicable to different types of robots. With this method, we fabricate two types of robot: a fish and a jellyfish (Fig. 1), and test them in water. The structure and actuation principle of the robots are explained in Section II. The fabrication process of the robots is detailed in Section III. Swimming demonstration and thrust measurement of the fabricated robots are performed in Section IV, followed by the discussion and conclusions in Section V.

\section{Structure AND ACtUATION PRINCIPLE}

DEAs consist of an elastomer membrane sandwiched between two compliant electrodes, as shown in Fig. 2(a). Applying a voltage between the electrodes leads to an attractive electrostatic force (Maxwell pressure), squeezing the membrane, resulting in thickness reduction and area expansion. The robots consist of several bonded layers of 
(a)
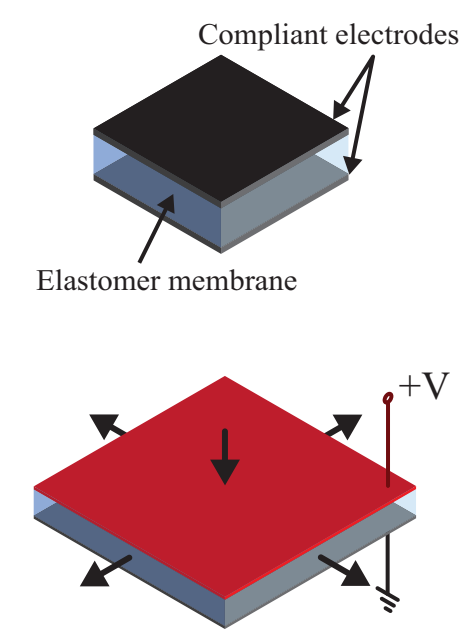

(b)

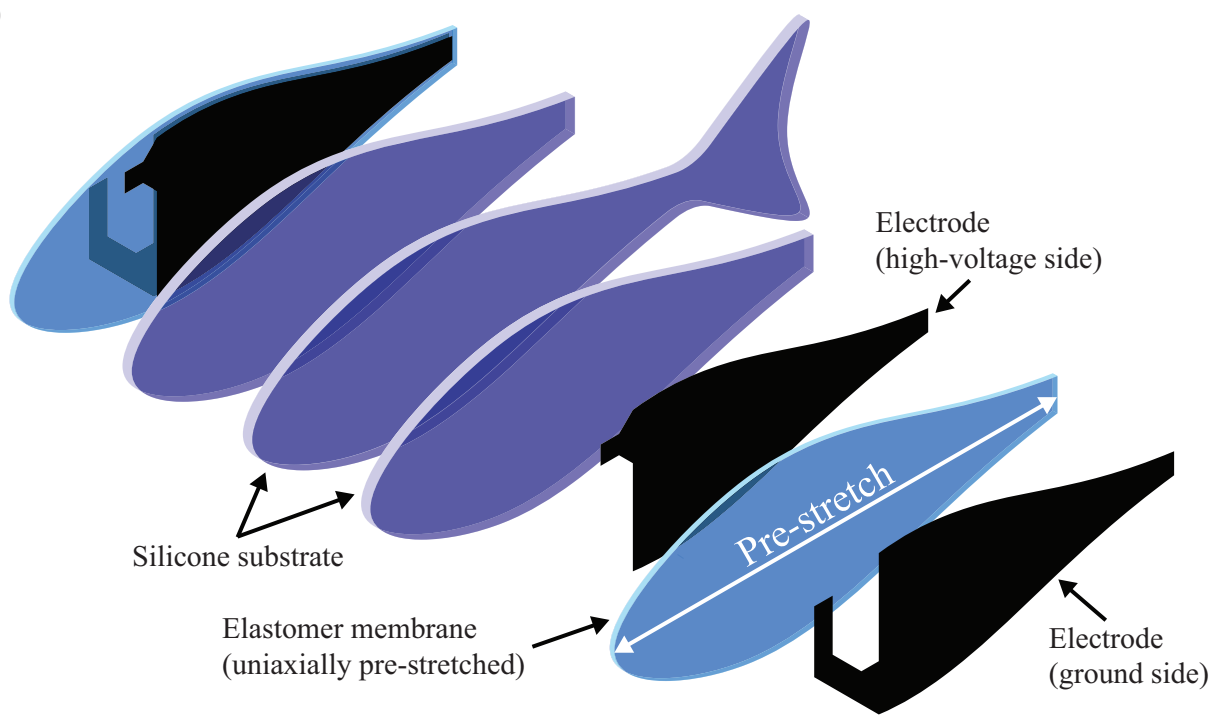

Fig. 2. (a) DEA actuation principle. DEAs consist of an elastomer membrane sandwiched between two compliant electrodes. Applying a voltage generates compressive electrostatic force, which squeezes the structure, leading to thickness reduction and area expansion. (b) Structure of the fish robot consisting of several silicone layers.

soft silicones. The silicone can serve as an insulator and a spring, or can be mixed with carbon black to form flexible electrodes. The robot structure has two main types of parts. One is the active part where the DEA electrodes overlap, and the other is passive part with only one or no electrodes. The active part induces movements of the passive part by the DEA actuation. Subsequently, the passive part generates thrust forces as the result of reaction forces to the surrounding water. In the robots, the active and passive parts correspond respectively to the body and tail in the fish robot, and the fingers and fins in the jellyfish robot. This principle, generation of thrust forces by deformation of passive part, is widely used in existing biomimetic underwater robots (e.g., [5], [12], [13]).

Fig. 2(b) shows the structure of the fish robot. Two DEAs pre-stretched uniaxially form an antagonistic configuration via the silicone substrates. The high-voltage electrode is slightly smaller than that of the silicone substrates and the elastomer membranes, so this electrode is completely insulated (i.e., there is no path of electrical short circuit via the surrounding water). This insulation adds nearly no stiffness to the robot. In the robot, one of the silicone substrates has the body-tail shape, while the others have only the body shape. This realizes fish-like bending stiffness distribution of the structure along the longitudinal direction; the bending stiffness is higher in the body, and lower in the tail [14]. The entire shape of the robot is similar to that of a horse mackerel (Carangidae family). The area of the electrodes is chosen to match the swimming muscles in fish which are distributed between the pectoral fin and the tail base, as can be seen in yellowtail kingfish (Seriola lalandi) in the family Carangidae [15]. When all the layers are assembled, the robot has a straight shape, as shown in

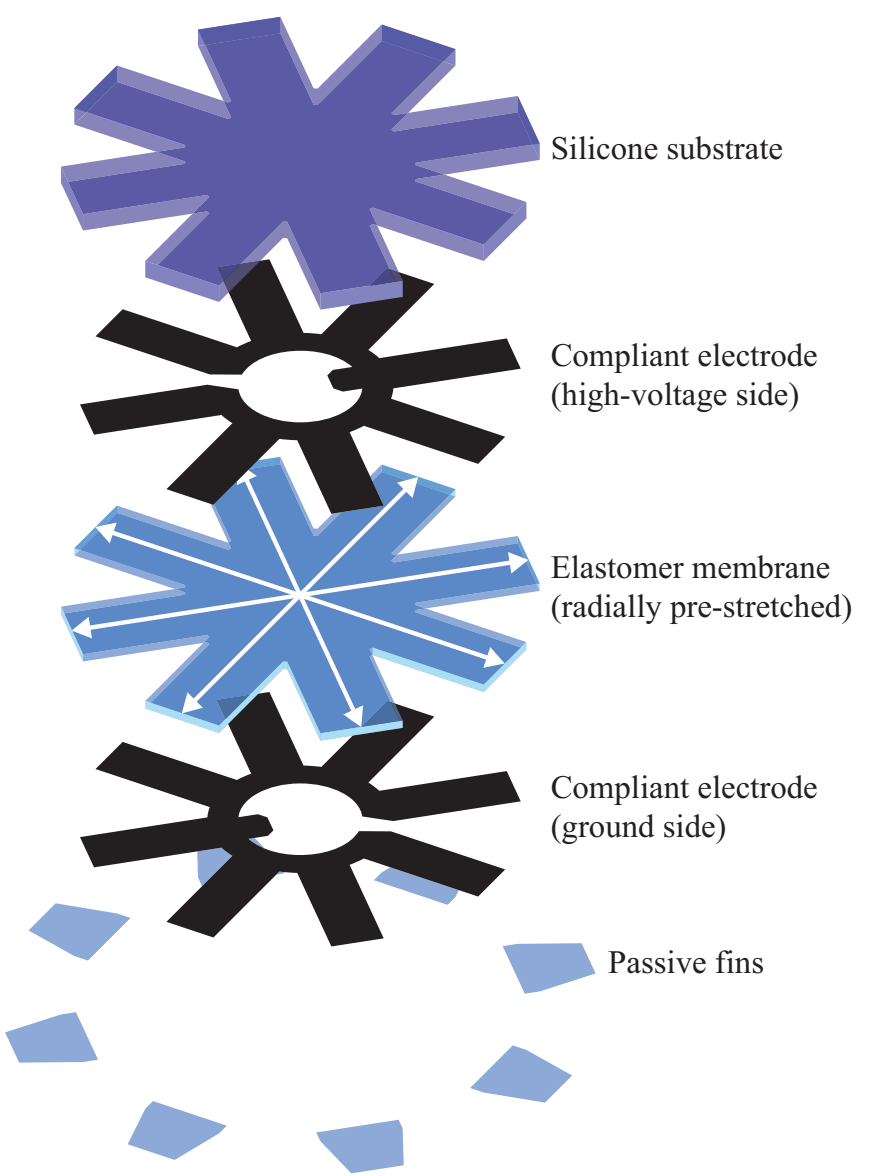

Fig. 3. Structure of the jellyfish robot consisting of several silicone layers.

Fig. 4(ai). Activating one of the DEAs (Fig. 4(aii)) induces its elongation, resulting in a bending motion of the body 
(a)

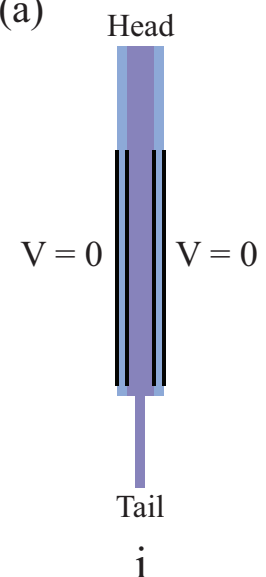

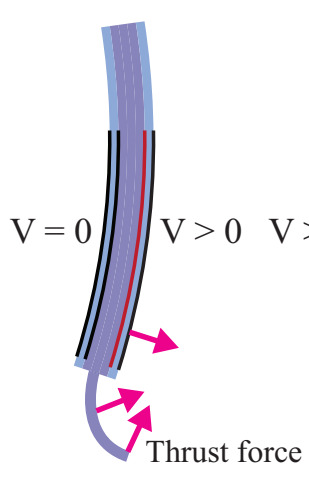

ii

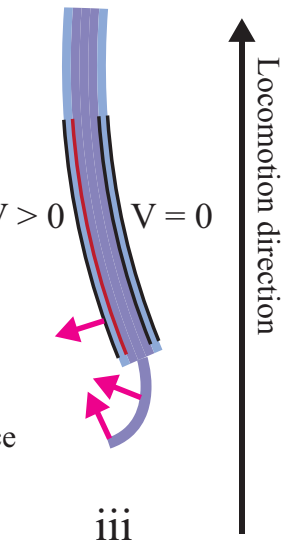

(b)

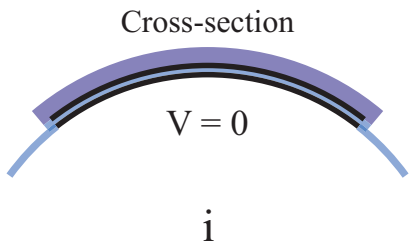

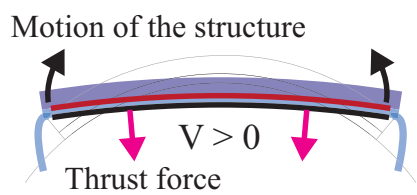

ii

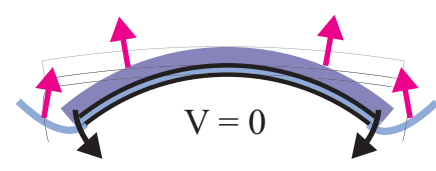

iii

Fig. 4. (a) Actuation principle of the fish robot. i: The robot has a straight shape in the initial unactuated state. ii: Activating one of the DEAs deforms the tail, generating thrust. iii: Periodic actuation of the DEAs one after the other between the states ii and iii generates thrust in the direction that moves the robot forward. (b) Actuation principle of the jellyfish robot. i: The robot has a bell shape in the initial state due to the pre-stretch of the DEA. ii: Activation of the DEA moves the fingers towards an overall flat shape. iii: Deactivation of the DEA moves back the structure toward the bell shape. When the states ii and iii are achieved one after the other periodically, the differential of the thrust forces moves the robot upward.

with largest amplitude at the tail base. At the same time, the tail is passively moved and deformed. The reaction forces on the tail and the body against the surrounding water result in thrust forces. Since the structure of the robot is symmetric with respect to the mid-body plane, activating the DEAs sequentially and periodically (between the states ii and iii in Fig. 4(a)) generates thrust pushing the robot forward.

The structure of the jellyfish robot is shown in Fig. 3. As for the fish robot, the electrodes are slightly smaller the silicone substrate and the elastomer membrane, to ensure high voltage insulation. Passive fins are attached at the tip of each finger. The DEA is radially pre-stretched so that after all the layers are bonded, the robot takes a jellyfish-like bell shape due to the pre-stretch, where the internal stress of the DEA and the bending moment of the silicone substrate are balanced, similar to the self-organized, dielectric elastomer minimum energy structure [16]. Fig. 4(bi) represents this state in cross-sectional view. When the DEA is activated, it reduces the internal stress and the fingers move toward a flat shape (Fig. 4(bii)). In this state, thrust forces are generated in the downward direction, as the result of the reaction to the surrounding water. Given their shape and attachment mechanism, the fins bend to approximately $90^{\circ}$ and contribute nearly no thrust. When the DEA is deactivated, the structure returns to the initial bell shape (Fig. 4(biii)), generating thrust forces in the upward direction. But now, the fins remain more horizontal, generating thrust. The passive fin motion gives an asymmetry in thrust between the opening and closing motions. When the robot is periodically actuated between states ii and iii, the differential of thrust forces results in the upward movement of the robot.

\section{FABRICATION}

We first explain the fabrication process of the fish robot represented in Fig. 5(a-e). Dimensions are given in mm. (a) The first steps was blade-casting a liquid silicone elastomer
(CF19-2186, NuSil Technology) on a polyethylene terephthalate (PET) sheet using a universal applicator (ZUA2000, Zehntner) and a film applicator (ZAA2300, Zehntner). After curing at $80{ }^{\circ} \mathrm{C}$ for $60 \mathrm{~min}$, a $80 \mu \mathrm{m}$-thick elastomer membrane was formed. (b) The membrane was then peeled off from the PET sheet, pre-stretched uniaxially with a ratio of 1.25 , and held in an acrylic (PMMA) holding frame using a silicone adhesive film (ARclear 8932EE, Adhesives Research). (c) Subsequently, compliant electrodes were patterned on the both sides of the membrane using a pad-printing method, forming the DEA. The composition of the electrodes and details of the pad-printing method are available in [10] and [17]. (d) $250 \mu$ m-thick silicone substrate (Sylgard 184, Dow Corning) was cut into the body shape by a $\mathrm{CO} 2$ laser cutter, and bonded on the DEA using oxygen plasma surface activation (Zepto plasma system, Diener electronic). A small amount of ethanol was placed between the DEA and the substrate prior to the bonding to remove bubbles trapped at the interface [18], ensuring the complete encapsulation of the high-voltage-side DEA electrode. To realize the electrical connection to this electrode, a punched hole was made. (e) Finally, two of the DEA parts fabricated in the previous step were attached on the both sides of a $500 \mu \mathrm{m}$-thick silicone substrate that had the body-tail shape. Rubber insulated wires were connected to the electrodes using a silver epoxy (ELECTON 40AC, Amepox), a conductive tape, and a silicone glue (Sylgard RTV-734, Dow Corning). The mass of the fabricated robot was $3.8 \mathrm{~g}$.

The fabrication process of the jellyfish robot represented in Fig. 5(f-i) followed essentially the same method and technology used for the fish robot. (f) a $80 \mu \mathrm{m}$-thick elastomer membrane (CF19-2186, NuSil Technology) was radially prestretched with a ratio of 1.2, and held in a PMMA holding frame. (g) After patterning the electrodes, a $600 \mu \mathrm{m}$-thick 
(a)

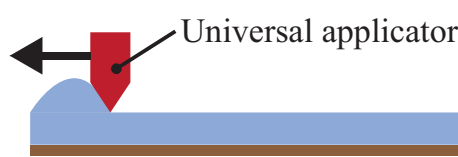

(b)

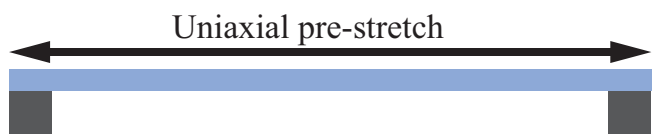

(c)

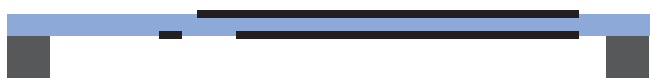

(d)

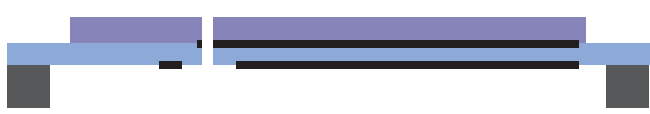

(e)

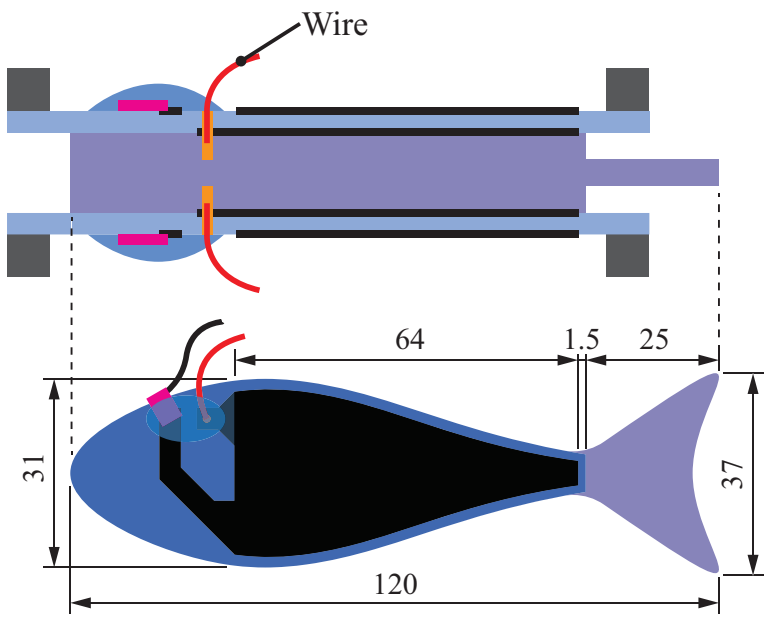

(f)

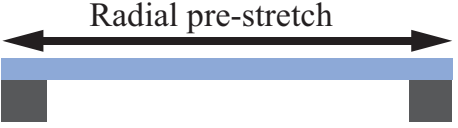

(g)

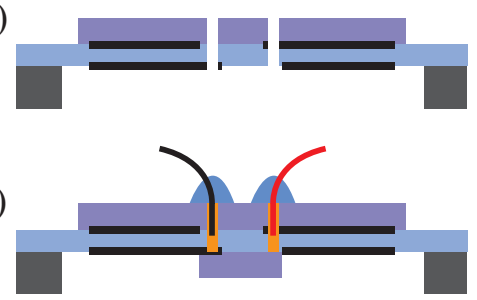

(i)

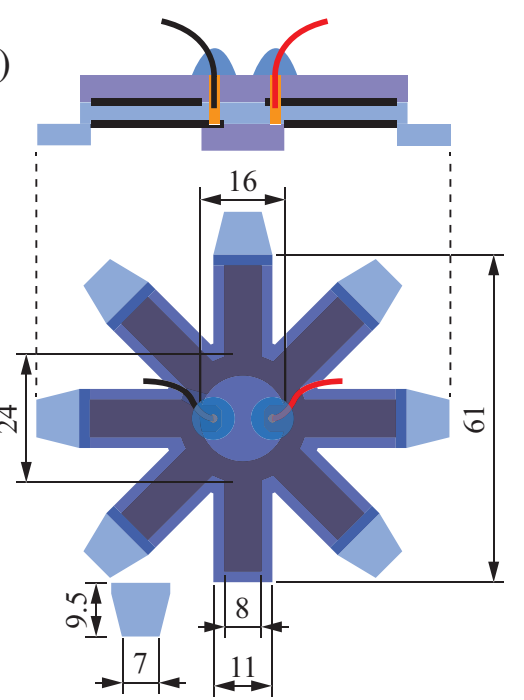

Elastomer membrane

PET

PMMA

Electrode

Silicone substrate

Conductive tape

Silicone glue

Silver epoxy

Fig. 5. Fabrication process of the robots. Dimensions are in mm. (a-e) represent the process for the fish robot. (a) Blade-casting and curing a liquid silicone elastomer (b) Pre-stretch the elastomer membrane. (c) Patterning the electrodes. (d) Bonding the silicone substrate on the membrane and making a punched hole to form the DEA part. (e) Bonding two of the DEA parts on the body silicone substrate, and finish wiring. The process of the jellyfish robot represented in (f-i) is fundamentally the same as the one used for the fish robot.

silicone substrate (Silbione LSR-4305, Bluestar Silicones) was bonded on the DEA, followed by punching the holes. (h) Subsequently, the electrical connections were made, while attaching another silicone substrate on the bottom to cover the holes. (i) The robot was then cut from the holding frame, and passive fins made of a $100 \mu \mathrm{m}$-thick elastomer membrane (Sylgard 184, Dow Corning) were attached on the finger tips. The mass of the fabricated robot was $2.6 \mathrm{~g}$. All the silicone membranes and substrates used were fabricated based on the setup and curing condition mentioned in the process step (a).

\section{Swimming Demonstration and Thrust MEASUREMENT}

Swimming demonstration of the fabricated robots was performed in a water tank filled with tap water. Fig. 6(a) shows the fish robot with floats (foam) and a weight (metal washer) attached for balance. Above the floats (i.e., water surface), the rubber insulated wires were connected to very thin enameled copper wires ( $36 \mu \mathrm{m}$ in diameter) to minimize the effect of wires stiffness on swimming speed. A similar approach was used for the jellyfish robot (except the weight). A CMOS camera was used to measure the locomotion speed. The robots were activated using high-voltage square waves generated from a computer running LabVIEW, a switching circuit, and a high-voltage power supply (PS350, Stanford Research Systems).

A sequence of images of the fish robot swimming is shown in Fig. 6(b), using a driving frequency of $3 \mathrm{~Hz}$ and an applied voltage of $3 \mathrm{kV}$, sequentially activating the two antagonistic DEAs. The periodic bending motion of the robot body passively deformed the tail, resulting in the forward movement. The measured locomotion speed of the robot was $\sim 8 \mathrm{~mm} / \mathrm{s}$ at the time section of $0-9 \mathrm{~s}$.

Fig. 6(c) shows the sequence of the jellyfish robot swimming, with a driving frequency of $2 \mathrm{~Hz}$ and an applied voltage of $3 \mathrm{kV}$. The passive deformation of the fins followed the periodic open-close motion of the fingers, and moved the robot toward the right side in the figure. The measured locomotion speed of the robot was $\sim 1.5 \mathrm{~mm} / \mathrm{s}$ in the entire time section. 

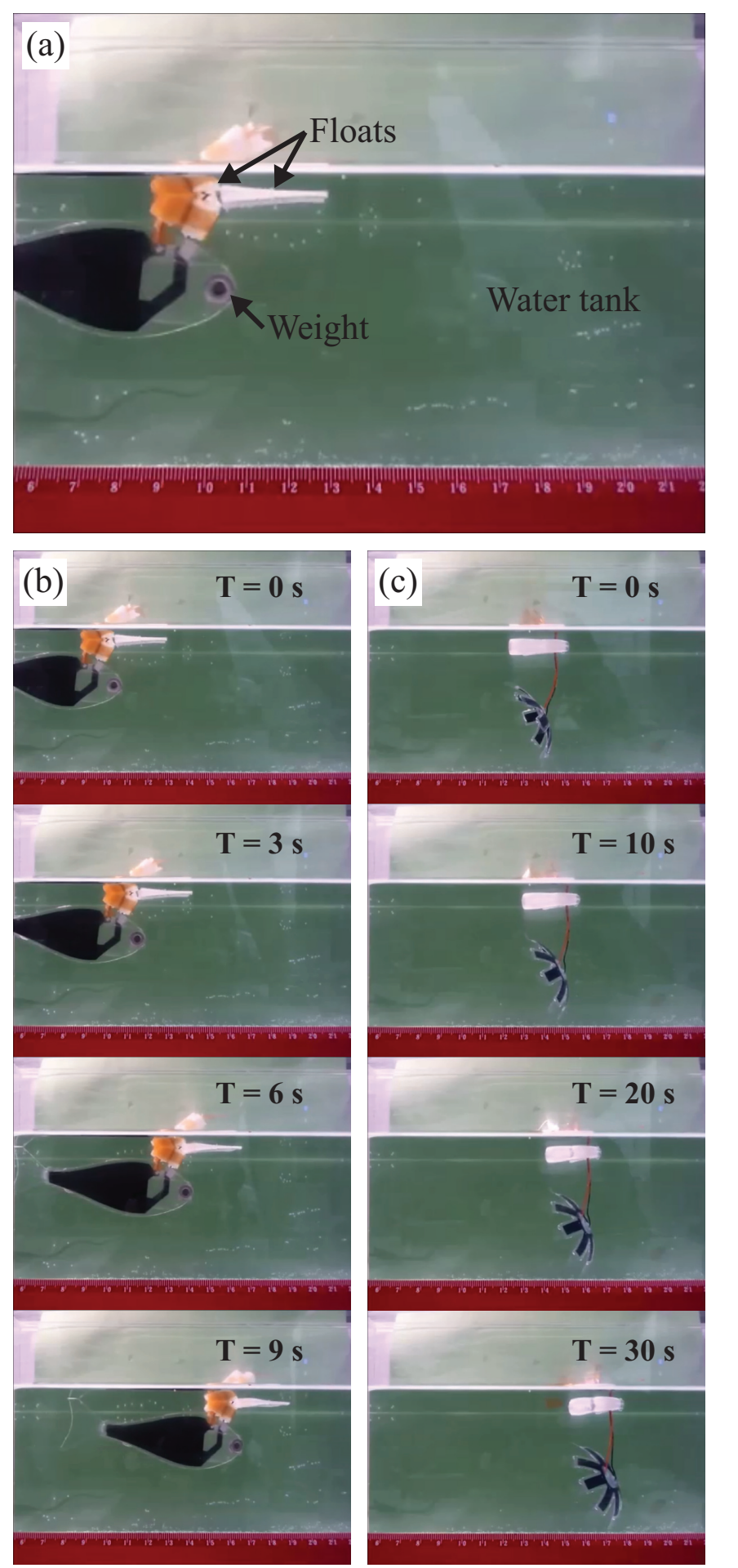

Fig. 6. Swimming demonstration of the fabricated robots. (a) Setup used for the demonstration. A water tank was filled with tap water. Floats and a weight were attached for balancing the robot. Electric wires were not visible above the floats, due to small diameter $(36 \mu \mathrm{m})$. (b) Sequence of the fish robot swimming with the driving frequency $3 \mathrm{~Hz}$ at $3 \mathrm{kV}$ of the applied voltage. The measured locomotion speed was $\sim 8 \mathrm{~mm} / \mathrm{s}$. (c) Sequence of the jellyfish robot swimming with the driving frequency $2 \mathrm{~Hz}$ at $3 \mathrm{kV}$ of the applied voltage. The measured locomotion speed was $\sim 1.5 \mathrm{~mm} / \mathrm{s}$.

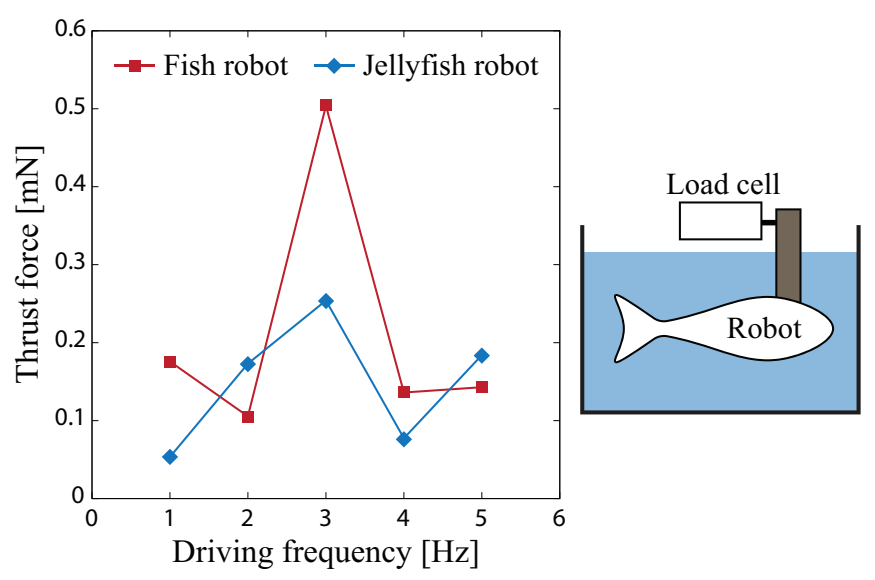

Fig. 7. Measured thrust force as a function of the driving frequency (applied voltage $3 \mathrm{kV}$ ).

The same water tank was used for thrust force measurements. A low-capacity load cell (UF1, Applied Measurement Limited) was connected to the robot via a probe, which also held the robot in place. The measured thrust vs. drive frequency is shown in Fig. 7 for an applied voltage of $3 \mathrm{kV}$. The values plotted are average of $5 \mathrm{~s}$ measurement. The fish robot showed a peak force $0.5 \mathrm{mN}$ at $3 \mathrm{~Hz}$. This may result from a structural vibration mode. Since the entire structure of the robot deforms when driven, a large amplitude of the tail was excited at particular frequencies, generating a large thrust. The data also suggested another resonance peak near $1 \mathrm{~Hz}$. The jellyfish robot showed a peak thrust of $0.25 \mathrm{mN}$ at $3 \mathrm{~Hz}$. The results obtained here represent the locomotion speed of each robot independently, because steady speed is given by the balance between the thrust and the drag [19]. This is the reason why the jellyfish robot is much slower than the fish one, even the thrust force is only roughly 2 times lower.

\section{DISCUSSION AND CONCLUSIONS}

We have demonstrated a method to create DEA-based simple biomimetic underwater robots, through the fabrication and the swimming tests of two types of robots: a fish and a jellyfish. There is significant potential to improve the robots swimming performance by optimizing mechanical parameters. Optimal pre-stretch and thickness of the DEAs increase actuation strokes and forces (e.g., [9], [20]), and hence speed and thrust. In the jellyfish robot, the pre-stretch also modifies the bell shape, linked to drag, and can lead to higher locomotion speed. Changing the thickness of the silicone substrates will change the bending stiffness, and can change stroke amplitude. Similarly, the geometry and stiffness of the passive parts are also important parameters to maximize thrust [21]. Further characterization of swimming speed, thrust, and tail amplitude as a function of the drive voltage and frequency should be performed for different device geometries. Modeling the robots can establish stronger design principles, using analytical and finite element methods 
based on hyperelastic material models that are often used for modeling DEAs (e.g., [9], [20]).

Our method, lamination of silicone layers, could enable a wide range of robots using different swimming principles, as classified in [1]. Examples include eel-like undulating robots and ray-like flapping robots. These robots could be realized in different sizes and geometries by scaling the laminated structure. Moreover, our method has the potential to achieve multidirectional movements to improve maneuverability of the robots. Two approaches can be considered for this purpose. One is to attach additional active fins to the robots. For example, the fish robot could be equipped with active pectoral fins. The other approach is segmentation of the DEA electrodes. This may enable, for instance, partial actuation of the jellyfish fingers to shift the direction of resulting thrust forces.

In the current fabrication process, thin, stretchable electrodes are patterned solely for the DEAs. This same electrode technology can also be used to integrate sensing parts as additional layers. Such sensing layers can be used to control the robots by measuring body deformations and water flow as resistance or capacitance changes. The sensing layers could also be included in passive parts such as tails and fins to characterize their complex deflection. In addition, DEAs can also be used as strain sensors (e.g., [22]).

Along with the sensing abilities, untethered locomotion could be achieved using miniaturized electronic components inclduing high-voltage DC/DC converters (e.g., Q series, EMCO [23], 2.05 cubic centimeters), lithium polymer (LiPo) batteries, and microcontroller boards. When these components are embedded, the robots should end up with a length of order $10 \mathrm{~cm}$. Given the power consumption of the converters and the DEAs, such untethered robots may be able to swim for a few hours with a 2 cell LiPo battery of $240 \mathrm{mAh}$. As reference point, a remotely controlled untethered airplane using DEAs for the active control surfaces can be seen in [24].

This study present a step to expand the use of DEAs in biomimetic underwater robots, and contributes to their future development.

\section{ACKNOWLEDGMENTS}

The authors would like to thank W. Conus, B. Grossniklaus, B. Schubert, S. Rosset, and S. Mintchev for their assistance and helpful discussions. This work was supported by the Swiss National Centre of Competence in Research (NCCR) Robotics, and the Swiss National Science Foundation grant 200020-153122.

\section{REFERENCES}

[1] M. Sfakiotakis, D. M. Lane, and J. B. C. Davies, "Review of fish swimming modes for aquatic locomotion," IEEE Journal of Oceanic Engineering, vol. 24, no. 2, pp. 237-251, 1999.

[2] S. Marras, and M. Porfiri, "Fish and robots swimming together: attraction towards the robot demands biomimetic locomotion," Journal of The Royal Society Interface, vol. 9, no. 73, pp. 18561868, 2012.

[3] I. D. Neveln, Y. Bai, J. B. Snyder, J. R.Solberg, O. M. Curet, K. M. Lynch, and M. A. MacIver, "Biomimetic and bio-inspired robotics in electric fish research," Journal of Experimental Biology, vol. 216, no. 13, pp. 2501-2514, 2013.
[4] W. Chu, K. Lee, S. Song, M. Han, J. Lee, H. Kim, M. Kim, Y. Park, K. Cho, and S. Ahn, "Review of biomimetic underwater robots using smart actuators," International Journal of Precision Engineering and Manufacturing, vol. 13, no. 7, pp. 1281-1292, 2012.

[5] A. D. Marchese, C. D. Onal, and D. Rus, "Autonomous soft robotic fish capable of escape maneuvers using fluidic elastomer actuators," Soft Robotics, vol. 1, no. 1, pp. 75-87, 2014.

[6] P. Brochu and Q. Pei, "Advances in dielectric elastomers for actuators and artificial muscles," Macromolecular Rapid Communications, vol. 31, no. 1, pp. 1036, 2010.

[7] I. A. Anderson, T. A. Gisby, T. G. McKay, B. M. OBrien, and E. P. Calius, "Multi-functional dielectric elastomer artificial muscles for soft and smart machines," Journal of Applied Physics, vol. 112, no. 4, pp. 041101-1-041101-21, 2012.

[8] F. B. Madsen, A. E. Daugaard, S. Hvilsted, and A. L. Skov, "The current state of silicone-based dielectric elastomer transducers," Macromolecular Rapid Communications, DOI: 10.1002/marc.201500576.

[9] S. Akbari, S. Rosset, and H. Shea, "Improved electromechanical behavior in castable dielectric elastomer actuators ", Applied Physics Letters, vol. 102, no. 7, pp. 071906-1-071906-5, 2013.

[10] L. Maffli, S. Rosset, M. Ghilardi, F. Carpi, and H. Shea, "Ultrafast all-polymer electrically tunable silicone lenses," Advanced Functional Materials, vol. 25, no. 11, pp. 16561665, 2015.

[11] H. Godaba, W. Ng, and J. Zhu, "Development of a soft jellyfish robot based on dielectric elastomer actuators," Workshop on soft robots, in IEEE International Conference on Robotics and Automation, Hong Kong, China, June 2014.

[12] P. V. y Alvarado, and K. Youcef-Toumi, "Design of machines with compliant bodies for biomimetic locomotion in liquid environments," Journal of Dynamic Systems, Measurement, and Control, vol. 128, no. 1, pp. 313, 2006.

[13] A. Villanueva, C. Smith, and S. Priya, "A biomimetic robotic jellyfish (Robojelly) actuated by shape memory alloy composite actuators," Bioinspiration \& Biomimetics, vol. 6, no. 3, pp. 036004-1-03600416, 2011.

[14] M. J. McHenry, C. A. Pell, and J. H. Long Jr., "Mechanical control of swimming speed: Stiffness and axial wave form in undulating fish models," Journal of Experimental Biology, vol. 198, pp. 2293-2305, 1995.

[15] K. Yanase, N. A. Herbert, and J. C. Montgomery, "Disrupted flow sensing impairs hydrodynamic performance and increases the metabolic cost of swimming in the yellowtail kingfish, Seriola lalandi," Journal of Experimental Biology, vol. 215, no. 22, pp. 3944-3954, 2012.

[16] G. Kofod, W. Wirges, M. Paajanen and S. Bauer, "Energy minimization for self-organized structure formation and actuation," Applied Physics Letters, vol. 90, no. 8, pp. 081916-1-081916-3, 2007.

[17] S. Rosset, S. Araromi, S. Schlatter, and H. Shea, "Fabrication process of silicone-based dielectric elastomer actuators," Journal of Visualized Experiments, no. 108, pp. e53423-1-e53423-13, 2016.

[18] S. Araromi, S. Rosset and, H. Shea, "High-resolution, large-area fabrication of compliant electrodes via laser ablation for robust, stretchable dielectric elastomer actuators and sensors," ACS Applied Materials \& Interfaces, vol. 7, no. 32, pp. 18046-18053, 2015.

[19] A. Azuma, The biokinetics of flying and swimming, 2nd ed., Virginia: American Institute of Aeronautics and Astronautics, 2006.

[20] B. OBrien, T. McKay, E. Calius, S. Xie, and I. Anderson, "Finite element modelling of dielectric elastomer minimum energy structures," Applied Physics A, vol. 94, no. 3, pp. 507-514, 2009.

[21] Y.-J. Park, U. Jeong, J. Lee, S.-R. Kwon, H.-Y. Kim, and K.-J. Cho, "Kinematic condition for maximizing the thrust of a robotic fish using a compliant caudal fin," IEEE Transactions on Robotics, vol. 28. no. 6, pp. 1216-1227, 2012.

[22] G. Rizzello, D. Naso, A. York, and S. Seelecke, "Closed loop control of dielectric elastomer actuators based on self-sensing displacement feedback," Smart Materials and Structures, vol. 25, no. 3, pp. 0350341-035034-13, 2016.

[23] EMCO High Voltage Corp., "EMCO proportional voltage products," [Online]. Available: http://www.emcohighvoltage.com/proportionalpower-supply.php.

[24] J. Shintake, S. Rosset, B. Schubert, D. Floreano and H. Shea, "A foldable antagonistic actuator," IEEE/ASME Transactions on Mechatronics, vol. 20, no. 5, pp. 1997-2008, 2015. 